\title{
Dynamic electronic compensation of fixed gratings in photorefractive media
}

\author{
Sergei Orlov and Demetri Psaltis \\ California Institute of Technology, Department of Electrical Engineering 116-81, Pasadena, \\ California 91125 \\ Ratnakar R. Neurgaonkar \\ Rockwell International Science Center, 1049 Camino dos Rios, Thousand Oaks, California 91360
}

(Received 26 May 1993; accepted for publication 24 August 1993)

We present a model describing the formation of a photorefractive grating in the presence of photorefractively inactive space-charge modulation. The treatment is based on standard linearized Kukhtarevs equations. The model developed is applied to the interpretation of experimental results on electrical fixing in $\mathrm{Sr}_{0.75} \mathrm{Ba}_{0.25} \mathrm{Nb}_{2} \mathrm{O}_{6}$.

We present a model that describes the formation of a photorefractive grating in the presence of a fixed grating. In general, fixing methods demonstrated in different photorefractive crystals ${ }^{1-7}$ create an optically unerasable charge or polarization distribution. The model predicts how the fixed grating affects the formation of the ordinary photorefractive grating and also leads to a spatially nonuniform mobile charge distribution even in the presence of a homogeneous illumination. There is good agreement between the predictions of the model and recent experimental results ${ }^{7}$ on electrical fixing of holograms in $\mathrm{Sr}_{0.75} \mathrm{Ba}_{0.25} \mathrm{Nb}_{2} \mathrm{O}_{6}$.

The dynamic behavior of the photorefractive effect is described by the following set of nonlinear coupled equations. ${ }^{8}$ We neglect the contribution of the photovoltaic effect and assume that only one type of carrier is involved:

$$
\begin{aligned}
& \frac{\partial N_{D}^{+}}{\partial t}=\left(\alpha I_{0}+\beta\right)\left(N_{D}-N_{D}^{+}\right)-\gamma_{e} n_{e} N_{D}^{+}, \\
& j_{e}=e \mu n_{e} E+k_{b} T \mu \nabla n_{e}, \\
& \frac{\partial n_{e}}{\partial t}=\frac{\partial N_{D}^{+}}{\partial t}+\frac{1}{e} \nabla j_{e}, \\
& \nabla\left(P_{s}+\epsilon \epsilon_{0} E\right)=e\left(N_{D}^{+}-n_{e}-N_{a}+\rho_{\mathrm{fix}} / e\right),
\end{aligned}
$$

where $N_{D}^{+}$is ionized donors concentration, $n_{e}$ the free electron concentration, $N_{a}$ the acceptor concentration, $\mu$ the carrier mobility, $\gamma_{e}$ the recombination constant, $P_{s}$ the spontaneous polarization, and $E$ the electric field. We consider a sinusoidal fixed charge distribution: $\rho_{\text {fix }}$ $=\rho_{\mathrm{fx} x_{1}} \exp (i K x)$ and spatially uniform light illumination $I_{0}$. Following the usual linearization procedure and assuming that $\rho_{\mathrm{fix}}<e N_{a}$, the quantities $N_{D}^{t}, n_{e}, E$, and $j_{e}$ can be approximated by a sinusoidal form: $F(x)=F_{0}$ $+F_{1} \exp (i K x)$. Assuming that $P_{s}$ is uniform, the steadystate solution of the Eqs. (1)-(4) for the space-charge field and the ionized donors density modulation is given by

$$
\begin{aligned}
& E_{1}=\frac{\rho_{\mathrm{fix}_{1}}}{i K \epsilon \epsilon_{0}} \frac{E_{d}-i E_{0}}{E_{d}+E_{q}-i E_{0}}, \\
& N_{D_{1}}^{+}=-\frac{\rho_{\mathrm{fix}_{1}}}{e} \frac{E_{q}}{E_{d}+E_{q}-i E_{0}},
\end{aligned}
$$

where $E_{d}=K k_{b} T / e$-diffusion field, $E_{q}=N_{E} e / \epsilon \epsilon_{0} K$ is the limiting space-charge field, and $E_{0}$ the external applied field. The time constant with which the field $E_{1}$ is formed is equal to the conventional grating formation time constant with the same average intensity and spatial frequency, formed in the absence of the fixed grating. ${ }^{9}$ This is because the fixed grating acts as a nonhomogeneous term for the system of linearized Eqs. (1)-(4) replacing the sinusoidal intensity illumination; the coefficients of the set of linear equation remain unchanged, and hence the time constant is the same.

The total space-charge field [Eq. (5)] may be split into a sum of a field $\rho_{\mathrm{tix}} / i K \epsilon \epsilon_{0}$ induced by fixed, nonphotorefractive charges and a compensating field $E_{1}^{\text {comp }}$ formed by redistributed charge carriers described by $N_{D_{1}}^{+}$. Namely,

$$
E_{1}^{\mathrm{comp}}=\frac{e N_{D_{1}}^{+}}{i K \epsilon \epsilon_{0}}=-\frac{\rho_{\mathrm{fix}}}{i K \epsilon \epsilon_{0}} \frac{E_{q}}{E_{d}+E_{q}-i E_{0}} .
$$

Note that due to the finite dark conductivity, compensation of the fixed grating by dynamic electronic space-charge takes place even when no homogeneous illumination is present.

Our description so far assumed a fixed grating due to an ionic grating. Similar analysis may be applied to the case where the fixed grating is produced by a spatial variation of the ferroelectric spontaneous polarization. A spatial variation of spontaneous polarization is formally equivalent to a fixed space-charge according to: ${ }^{10} \rho_{\text {ind }}=-\nabla P_{s}$. Then, assuming a sinusoidal modulation of spontancous polarization: $P_{s}=P_{s_{0}}+P_{s_{1}} \exp (i K x)$, Eq. (4) takes the form

$$
\frac{\partial E}{\partial x}=\frac{e}{\epsilon \epsilon_{0}}\left(N_{D}^{+}-n_{e}-N_{a}-\frac{1}{e} \frac{\partial P_{s}}{\partial x}\right)
$$

or, in linearized form, for the case $E_{0}=0$ :

$$
i K E_{1}=\frac{e}{\epsilon \epsilon_{0}}\left(N_{D}^{+}-n_{e_{1}}-\frac{i K}{e} P_{s_{1}}\right),
$$

where $\epsilon$ is the mean value of de dielectric constant. The value of the total space-charge field $E_{1}$ and of the compensating electronic component $E_{1}^{\text {comp }}$ are given by 


$$
\begin{aligned}
& E_{1}=-\frac{\vec{P}_{s_{1}}}{\epsilon \epsilon_{0}} \frac{1}{1+E_{q} / E_{d}}=-\frac{P_{s_{1}}}{\epsilon \epsilon_{0}} \frac{1}{1+\left(\Lambda / \Lambda_{e}\right)^{2}}, \\
& E_{1}^{\mathrm{comp}}=\frac{P_{s_{1}}}{\epsilon \epsilon_{0}} \frac{E_{q} / E_{d}}{1+E_{q} / E_{d}}=\frac{P_{s_{1}}}{\epsilon \epsilon_{0}} \frac{\left(\Lambda / \Lambda_{e}\right)^{2}}{1+\left(\Lambda / \Lambda_{e}\right)^{2}},
\end{aligned}
$$

where $\Lambda=2 \pi / K$ is the grating spacing and $\Lambda_{e}$ $=2 \pi \sqrt{\epsilon \epsilon_{0} k_{b} T / e^{2} N_{E}}$ is the Debye length.

The perturbation of the refractive index arises through the ordinary linear electro-optic effect due to the total space-charge field $E_{1}$. Even though it is more correct to describe the refractive index variation in terms of polarization rather than electric field, ${ }^{11}$ both approaches lead to the same result and, in this sense, are equivalent. The modulation in spontaneous polarization also causes spatial variations of the linear EO coefficient and the dc dielectric constant. However, for the case of zero applied field considered here these terms contribute only to the higher spatial orders of the index of refraction variation. Therefore, they do not affect the fundamental harmonic that is responsible for the formation of the hologram.

In the case of fixing via ionic compensation the amplitude of the compensating electronic grating is difficult to measure independently, since the thermal erasure of the ionic grating also perturbs the electronic pattern. Electrical fixing that involves ferroelectric polarization, on the other hand, enables one to perform direct observations and measurements of the dynamic compensating grating, since the polarization pattern can be erased simply by applying a strong positive electric field along the direction of spontaneous polarization of the photorefractive crystal. ${ }^{6,7}$ The latter process does not affect the compensating field $E_{1}^{\text {comp }}$, whose strength can then be determined by measuring the diffraction efficiency of the residual hologram.

We studied experimentally the process of fixing and compensation of phase holograms in photorefractive $\mathrm{Sr}_{0.75} \mathrm{Ba}_{0.25} \mathrm{Nb}_{2} \mathrm{O}_{6}$. Electrical fixing was achieved by applying a negative voltage pulse along the crystal $c$ axis after recording a space-charge grating with two input beams. ${ }^{7}$ To avoid beam coupling during recording and reconstruction, the ordinarily polarized beams were used and the holographic diffraction efficiency (HDE) was monitored by an extraordinarily polarized low-power $\mathrm{He}-\mathrm{Ne}$ laser beam Bragg matched to the initial hologram. A typical cycle of recording, fixation, optical and electrical erasure is shown on Fig. 1. After the fixed hologram reaches steadystate under illumination by a non-Bragg-matched erasing beam, a strong positive voltage pulse is applied erasing the polarization grating. The residual revealed hologram corresponds to the electronic compensating grating, which is measured by recording the power of light diffracted from it. Figure 2 shows the dependence of the steady-state values of the fixed HDE and the compensating revealed grating HDE with the grating spacing $\Lambda$. In agreement with the model proposed the amplitude of the electronic compensating grating [Eq. (11)] dramatically increases with grating spacing.

Since in our experiments the effects associated with beam coupling (i.e., longitudinal change of modulation in-

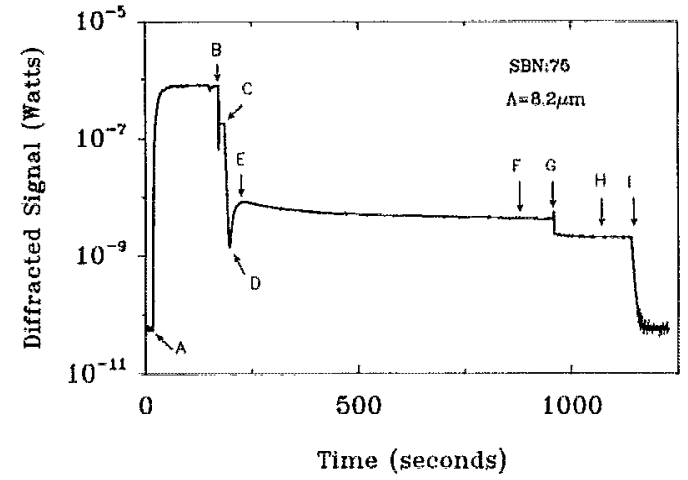

FIG. 1. Diffracted signal as a function of time during the fixing experiment. Fixing pulse amplitude is $-1650 \mathrm{~V} / \mathrm{cm}$ and its duration $0.5 \mathrm{~s}$. Total intensity of recording beams is $4 \mathrm{~mW} / \mathrm{cm}^{2}, \lambda=488 \mathrm{~nm}$, intensities ratio $m \approx 1$. The transmitted probe light power without the grating is $2.3 \mu \mathrm{W}$. A: Recording beings. B: Writing beams are blocked and a negative voltage pulse is applied. C: Optical erasure with a non-Bragg-matehed beam $\left(I=8 \mathrm{~mW} / \mathrm{cm}^{2}\right)$ begins. D: The transient dip due to $180^{\circ}$ phase shift between the polarization and the electronic gratings. E: Peak in HDE of the fixed grating; slow decay of fixed grating begins. F: Steady state of the fixed grating. G: Positive voltage pulse ( $E=4.2 \mathrm{kV} / \mathrm{cm}$, duration $2 \mathrm{~s}$ ) is applied (erasing beam is blocked). H: Revealed compensating grating. I: Optical erasure.

dex of hologram, self-depletion, or self-enhancement) were carefully climinated, the diffraction efficiency, in the limit of negligible absorption and small index variation, may be expressed as

$$
\eta \simeq\left(\frac{\pi d /\left|\Delta n_{1}\right|}{\lambda \cos \theta}\right)^{2} \propto r_{\mathrm{eff}}^{2} E_{1}^{2}
$$

where $d$ is the crystal thickness, $\theta$ is the Bragg angle inside the crystal, $r_{\text {eff }}$ is the effective linear $E O$ coefficient, and $E_{1}$ is the internal space-charge field variation. Then, according to Eqs. (10) and (11), independent of the actual value of the polarization modulation, the ratio of HDEs of the fixed and compensating (or "revealed") gratings should obey the following relationship:

$$
\ln \frac{\eta^{\mathrm{connp}}}{\eta^{\mathrm{fix}}}=4 \ln \Lambda / \Lambda_{e}+2 \ln r_{\mathrm{eff}}^{0} / r_{\mathrm{eff}}^{*},
$$

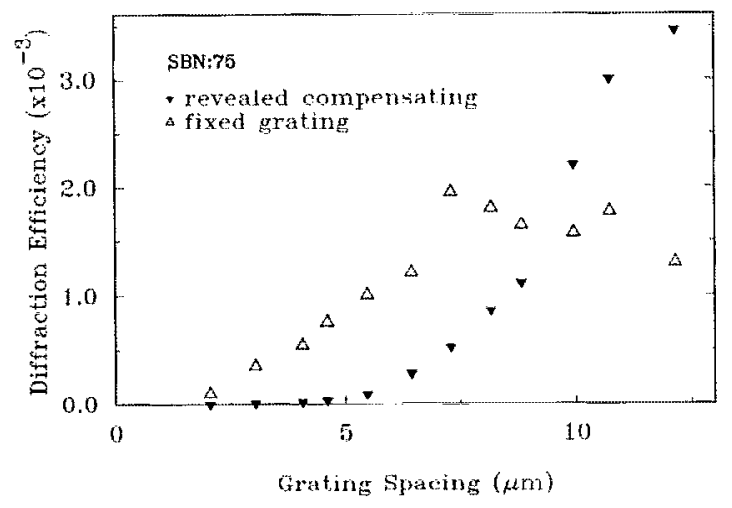

FIG. 2. Diffraction efficiencies of fixed (steady state) and electrically revealed compensating gratings as a function of $\Lambda$. The same experimental parameters were used as in Fig. 1. 


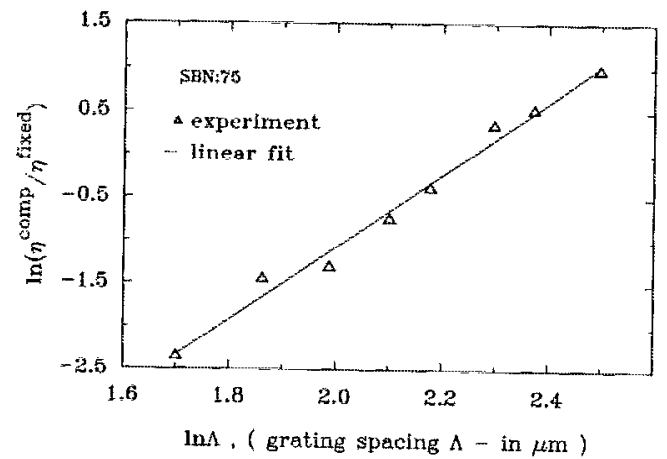

FIG. 3. Log-log plot of the ratio of HDEs of the revealed compensating grating and the fixed grating vs grating spacing. Points obtained for $A<5.5 \mu \mathrm{m}$ were not included since the diffracted signal off the revealed hologram is less than the noise level. The linear fit has the slope $4.2 \pm 0.2$ which is in good agreement with the theoretically predicted value 4.0.

where $r_{\text {eff }}^{*}$ is effective electro-optic coefficient of the partially depoled crystal after a negative depoling voltage is applied. The strong positive pulse that is applied to erase the polarization grating and to reveal the compensating grating also poles the crystal and, consequently, the $E O$ coefficient takes its original value $r_{\text {eff }}^{0}$. The experimental data shown in Fig. 3 indicate a linear relationship between $\ln \left(\eta^{\mathrm{comp}} / \eta^{\mathrm{fix}}\right)$ and $\ln \Lambda$ with slope $\simeq 4.2 \pm 0.2$ which is in a good agreement with the value 4 , predicted by the theory [Eq. (13)]. We should point out that since we do not know the dependence of $P_{s_{1}}$ with $\Lambda$, the fact that the ratio plotted in Fig. 3 is independent of $P_{s_{1}}$ makes possible the comparison between theory and experiment. The ratio of the HDEs of the compensating and fixed gratings is the same for both an ionic or a polarization fixed grating. However, the fact that we could observe an optically erasable re- vealed compensation grating by electrically erasing the fixed grating strongly favors the hypothesis that the fixing was due to polarization switching in the $\mathrm{Sr}_{0.75} \mathrm{Ba}_{0.25} \mathrm{Nb}_{2} \mathrm{O}_{6}$ fixing experiment. ${ }^{6,7}$ The maximum modulation depth of the spontaneous polarization, corresponding to the maximum fixed grating HDE, was evaluated to be equal to $\Delta P_{s} \simeq 10^{-4} P_{s}$, where $P_{s} \simeq 8.1 \mu \mathrm{C} / \mathrm{cm}^{2}$ is the spontaneous polarization of the completely poled $\mathrm{Sr}_{0.75} \mathrm{Ba}_{0.25} \mathrm{Nb}_{2} \mathrm{O}_{6}$ crystal at room temperature. ${ }^{6}$ This small modulation depth is responsible for the weak fixed holograms.

In conclusion, we have presented a model for the formation of a grating in the presence of photorefractively inactive, fixed charge, or polarization modulation. Application of this model to electrical fixing in $\mathrm{Sr}_{0.75} \mathrm{Ba}_{10.25} \mathrm{Nb}_{2} \mathrm{O}_{6}$ gives good agreement between experimental observations and theoretical predictions.

This work was supported by the Advanced Research Project Agency and the U.S. Air Force Office of Scientific Research. We thank Yong Qiao, Whye-Kei Lye, and Jiafu Luo for their help.

'J. J. Amodei and D. L. Staebler, Appl. Phys. Lett. 18, 540 (1971).

${ }^{2}$ D. Kirillov and J. Feinberg, Opt. Lett. 16, 1520 (1991).

${ }^{3}$ G. Montemezzani and P. Gunter, J. Opt. Soc. Am. B 7, 2323 (1990).

${ }^{4}$ L. Arizmendi, J. Appl. Phys. 65, 423 (1989).

${ }^{5}$ V. Leyva, A. Agranat, and A. Yariv, Opt. Lett. 16, 554 (1991).

${ }^{6}$ F. Micheron and G. Bismuth, Appl. Phys. Lett. 23, 71 (1973).

${ }^{7}$ Y. Qiao, S. Orlov, D. Psaltis, and R. Neurgaonkar, Opt. Lett. 18 (1993).

${ }^{8}$ N. Kukhtarev, V. Markov, S. Odoulov, M. Soskin, and V. Vinetskii, Ferroelectrics 22, 949 (1979).

${ }^{9}$ G. C. Valley and J. F. Lam, in Photorefractive Materials and Their Applications $I$, edited by P. Gunter and J. P. Huignard (Springer, Berlin, 1988), Chap. 3.

${ }^{10}$ L. D. Landau and E. M. Lifshitz, Electrodynamics of Continuous Media (Addison-Wesley, Reading, MA, 1960), Chap. 2.

${ }^{11}$ W. D. Johnston, Jr., J. Appl. Phys. 41, 3279 (1970). 